\title{
环糊精-石墨烯超分子体系
}

\author{
孙 涛 $^{a} \quad$ 李建业 ${ }^{b}$ 郝爱友 ${ }^{*, a}$ \\ $\left({ }^{a}\right.$ 山东大学化学与化工学院 济南 250100) \\ ( ${ }^{b}$ 滩坊科技学院 滩坊 262700)
}

\begin{abstract}
摘要 环糊精是由 $D$ 型吡喃葡萄糖通过 $\alpha-1,4-$ 糖苗键连接而成的环状超分子主体化合物, 其特殊的结构赋予了其良好 的分子识别性能; 石墨烯是仅由单层 $\mathrm{sp}$ 杂化的碳原子构筑的具有良好的电化学性能的材料. 作为著名的 “明星分子”, 石墨烯类材料无疑是近 5 年来研究热点之一. 在各种各样的石墨烯材料中, 由环糊精一石墨烯联合构筑的超分子体系在 保留二者优良性能的同时又引入了新的功能特点. 综述了近些年来新发展起来的环糊精-石墨烯超分子体系: 通过二 者作用方式进行了分类, 分为共价键连接和非共价键连接; 综述了该超分子体系在药物运输及释放、电化学检测(包括 对药物分子、污染物和生物分子的检测)等领域的应用; 最后对该体系在药物负载及释放、模拟生物固氮、燃料电池、 研究电子传导等应用前景进行了展望.
\end{abstract}

关键词 环糊精; 石墨烯; 超分子化学; 药物运输; 电化学检测

\section{Cyclodextrin-Graphene Supramolecular System}

\author{
Sun, Tao ${ }^{a}$ \\ Li, Jianye ${ }^{a}$ \\ Hao, Aiyou ${ }^{*, a}$ \\ ( ${ }^{a}$ School of Chemistry \& Chemical Engineering, Shandong University, Jinan 250100) \\ ( ${ }^{b}$ Chemical Engineering Department, Weifang University of Science and Technology, Weifang 262700)
}

\begin{abstract}
Cyclodextrins, composed by $D$-glucose units linked by $\alpha$-1,4-glucose bonds, have excellent molecule recognition ability for their particular structures. Graphene is a class of materials with good electrochemistry properties composed by one-atom-thick planar sheets of sp-bonded carbon atoms. As a famous "rising star", graphene is the hot topics in recent five years. Among the various graphene materials, cyclodextrin-graphene supramolecular systems can combine their advantages and also introduce novel properties. This paper reviews the recent research condition of the cyclodextrin-graphene supramolecular systems: the system is classified into covalently-bonded and non-covalently-bonded systems for the interaction modes; The applications in drug carrying \& release and electrochemistry detection (including drug molecules, pollutant and biomolecules) are reviewed; The prospects in the area of drug loading and release, mimic of bio-nitrogen-fixation-process, fuel cell and electron conducting study are raised.
\end{abstract}

Keywords cyclodextrin; graphene; supramolecular chemistry; drug transport; electrochemistry detection

超分子化学是近些年来研究热点之一. 超分子化学 可定义为 “超越分子的化学”, 是关于若干化学物种通 过分子间相互作用，包括氢键、疏水作用、静电作用、 $\pi-\pi$ 堆积作用等结合在一起所构成的具有一定组织性和功 能性、同时具有高度复杂性的整体的化学 ${ }^{[1]} .1987$ 年, Pederson ${ }^{[2]}, \mathrm{Cram}^{[3]}$ 和 $\mathrm{Lehn}^{[4]}$ 由于对超分子化学领域的 杰出贡献而获得该年度的诺贝尔化学奖. 在获得诺贝尔 奖 25 年来, 超分子化学并未像其他获得诺贝尔奖领域 一样出现没落的迹象, 反而发展得更加迅猛, 交叉材料 学、物理学、生物学甚至医学等学科, 大有成为 “超分 子科学” 的趋势.
石墨烯是被认为最有希望取代电子元件中硅的材 料, 自从 2004 年被发现之日起 ${ }^{[5]}$, 由于其优良的电化 学 ${ }^{[6-9]}$ 、热学 ${ }^{[10]}$ 和机械性能 ${ }^{[11,12]}$, 就注定其是一类 “明 星” 材料并吸引了大量关注的目光. 作为由碳组成的一 种结构, 石墨烯不单单是厚度达到前所未有的小, 而且 强度也是非常高, 并且同时还具有和铜一样的良好导电 性，在导热方面更是超越了目前已知的其他所有材料. 另外石墨烯近乎完全透明, 但其原子排列之紧密, 却连 具有最小气体分子结构的氦都无法穿透它. 碳, 这个地 球生命的基本组成元素, 再次让世人吃惊. 英国曼彻斯 特大学的 Andre Geim 和 Konstantin Novoselov 就是由于

*E-mail: haoay@sdu.edu.cn

Received May 17, 2012; revised June 5, 2012; published online June 13, 2012. 
对石墨烯材料开创性的研究而获得了 2010 年诺贝尔物 理学奖. 仅仅在石墨烯被发现 6 年就有科学家因此获得 诺贝尔奖, 这都充分证明了石墨烯作为继一维碳纳米 管、三维富勒烯之后的二维碳材料的重要科学意义.

石墨烯又名 “单层石墨片”，但是由于本身的结构 原因 $(\pi-\pi$ 堆积、疏水-疏水作用)使其趋于堆积成自组装 体, 而石墨烯的许多优良性质却只能在其以单层的二维 结构时才能展现出来 ${ }^{[13]}$, 这就使防止石墨烯自堆积成 为一个重要的课题.

环糊精(cyclodextrin, 简称 $\mathrm{CD}$ ) 是由 6,7 或 8 个 $D$ 吡喃葡萄糖单元通过 $\alpha-1,4$ 糖苷键连接而成的腔内疏 水、腔外亲水的环状低聚糖 ${ }^{[14]}$ (图 1), 分别称为 $\alpha-, \beta$-和 $\gamma$-环糊精. 环糊精是一种典型的无底无盖的 “锥筒” 状 分子, 由于其特殊的结构使其在水溶液中可以识别和包 结一系列的底物. 由葡萄糖单元构成, 使其成为超分子 化学中一种便宜易得、生物相容性良好的重要主体分子,

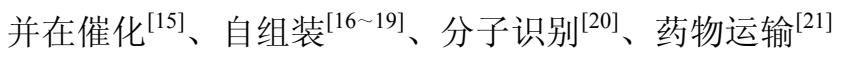
等领域应用广泛. 著名科学家徐光宪院士 ${ }^{[22]}$ 曾特别指 出: 环糊精超分子科学是 21 世纪化学领域 11 个突破口 之一. 如果将环糊精和石墨烯联系起来, 在达到增强石 墨烯水溶性并防止其自聚集的目的的同时, 还能将环糊 精与石墨烯优良分子性能结合在一起, 将达到 $1+1>2$ 的效果. 本文将按此超分子体系中环糊精-石墨烯的制 备表征和应用来分类, 其中制备表征又以作用模式(包 括共价键连接和非共价键连接) 不同分类来分别阐述.

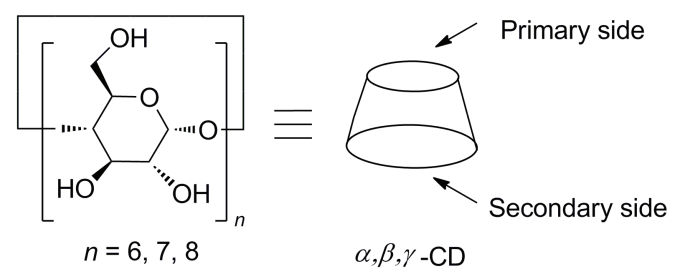

图 1 环糊精结构图

Figure 1 Structure of cyclodextrins

\section{1 环糊精-石墨烯超分子体系的制备与表征}

\section{1 共价键连接的环糊精一石墨烯超分子体系}

利用共价键连接的环糊精-石墨烯超分子体系具有 较强的化学稳定性, 有比较明确的化学组成, 目前的主 要合成途径还是利用氧化石墨烯与修饰的环糊精进行 共价键偶联.

石墨烯本身是一种相对 “巨大” 和有一定柔性的二 维材料, 具有构筑凝胶体系的潜力. 在提高水溶性的同 时, 将二维的石墨烯作为凝胶因子构筑三维的空间网格
体系将可能大大拓展石墨烯的应用范围，在此领域， Jiang 等 ${ }^{[23]}$ 做了有益的尝试(图 2). Jiang 等通过单-6-脱 氧一乙二胺- $\beta$-环糊精 $(\mathrm{EDA}-\mathrm{CD})$ 与氧化石墨烯反应得到 环糊精修饰的石墨烯片，进而通过超分子包合作用与偶 氮苯基团封端的嵌段共聚物聚-( $N, N$-二甲基丙烯酰 胺)- $b$-聚( $N$-异丙基丙烯酰胺) [ $a z o-(P D M A-b$-PNIPAM) $]$ 复合得到杂化的石墨烯环糊精客体包合物 (hybrid graphene inclusion complex, HGIC). 由于聚 $(N$-异丙基丙 烯酰胺)部分的热敏感性, HGIC 的水溶液在温度升高时 会发生溶胶一凝胶转变. 这种无机一有机杂化水凝胶是一 种有潜力的药物类小分子储存材料或能量转化材料. 另 外, 先利用强氧化剂将石墨烯氧化 (graphene oxide, GO), 然后通过共价键修饰石墨烯以实现其功能化, 目前看来 是得到石墨烯衍生物的主要要途径之一.

Wang 等 ${ }^{[24]}$ 利用微波催化的方法合成了新型环糊 精-石墨烯超分子体系: 先将石墨烯氧化物和羟丙基- $\beta$ 环糊精在超声处理下分散到去离子水中得到悬浊液, 然 后加热并利用微波辐射处理, 并加入水合肼和氨水还 原. 离心后用无水乙醇洗去未反应的羟丙基- $\beta$-环糊精, 干燥后即得环糊精一石墨烯超分子体系. 在此体系中, 环糊精和石墨烯是通过酯键相连接. 得到的环糊精一石 墨烯超分子体系具有较高的比表面积和分子识别能力, 有望于应用在富集和识别某些特定无机或有机分子领 域上.

吓啉(porphyrin)是一类由 4 个吡咯类亚基的 $\alpha$-碳原 子通过次甲基桥 $(=\mathrm{CH})$ 互联而形成的大分子杂环化合 物. 卟啉广泛存在于生命体中并有着重要的生理学意 义. 卟啉是一个高度共轭的体系, 并有良好的给电子能 力. 对基于卟啉和碳纳米管的光诱导电子转移过程和电 化学催化过程的研究 ${ }^{[25 ~ 28]}$ 已是当前研究热点之一. 把 卟啉引入到环糊精一石墨烯超分子体系中, 无疑加入了 更有利的元素，丰富和提高了环糊精-石墨烯超分子体 系的性质并拓展了其相应的应用范围. Wang 等 ${ }^{[29]}$ 通过 差丙基- $\beta$-环糊精和亚硫酰氯修饰的石墨烯氧化物之间 的亲核加成反应，然后利用嗍氢化钠在碱性条件下还原 得到了共价键连接的环糊精一石墨烯超分子体系(图 3), 利用红外吸收光谱、拉曼光谱及热重分析确认二者连接 基团为酯基. 利用苂光吸收光谱研究了此体系与四苯基 卟啉(tetra-phenyl-porphyrin, TPP)之间光诱导的电子转 移过程. 在 TPP 的 $N, N$-二甲基甲酰胺溶液中加入羟丙 基- $\beta$-环糊精会出现明显的苂光湮灭现象，而当加入石 墨烯氧化物或未经还原的环糊精一石墨烯氧化物连接体 时 TPP 的荧光强度却几乎不变, 这是与还原后得到的环 糊精-石墨烯超分子体系拥有更好的导电性能有关. 

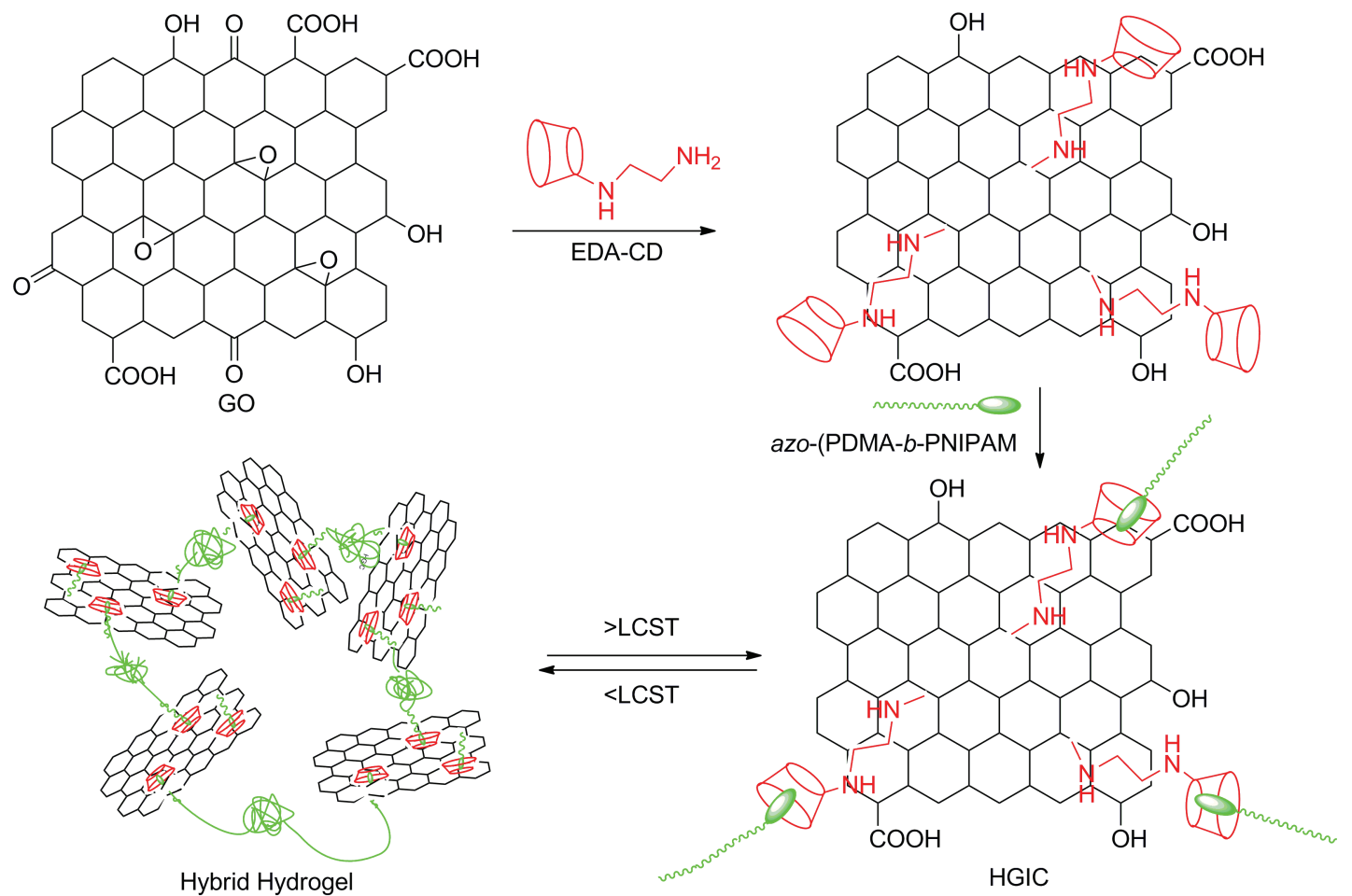

图 2 基于环糊精一石墨烯的凝胶

Figure 2 Gel based on cyclodextrin-graphene

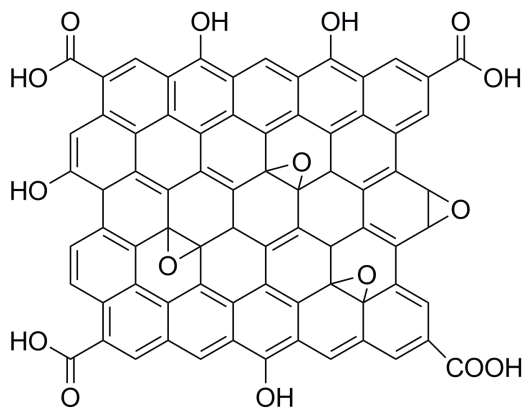

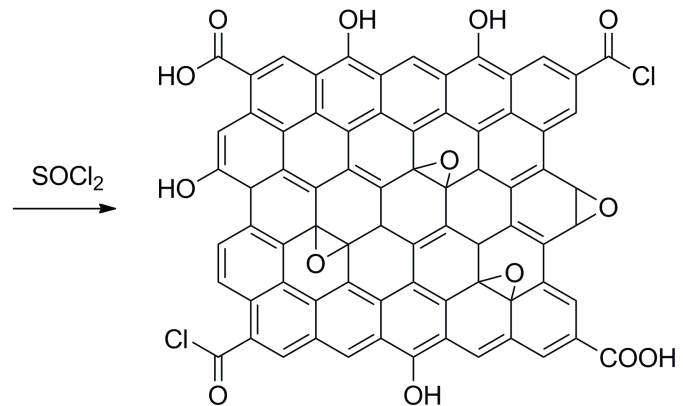
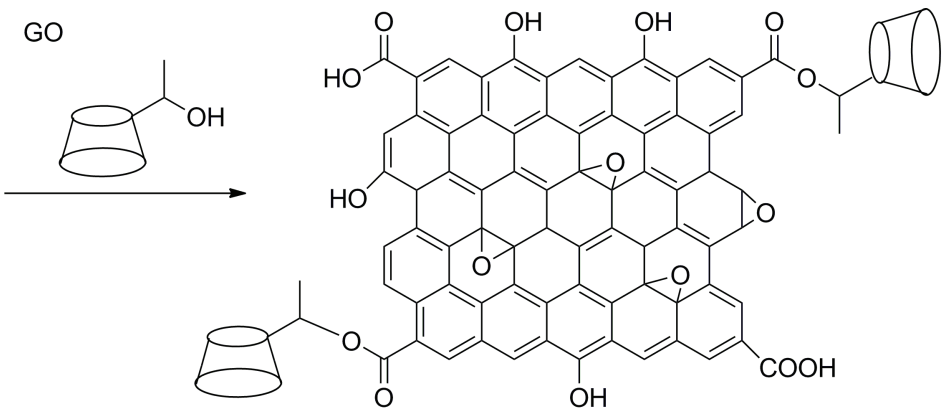

图 3 酯键连接的环糊精一石墨烯体系

Figure 3 Cyclodextrin-graphene system linked by easter groups

\section{2 非共价键连接的环糊精-石墨烯超分子体系}

非共价键作用环糊精一石墨烯超分子体系制备方法 简便，是目前环糊精一石墨烯超分子体系的主要制备手 段. 通过发展新型环糊精-石墨烯一卟啉超分子体系, Liu 等 ${ }^{[30]}$ 提出了新的药物携带与靶向运输模式: 修饰有金
刚烷基团的卟啉分子(2)作为连接体，将负载有抗癌药 物阿霉素(doxorubicin, DOX)的氧化石墨烯与叶酸基团 修饰的 $\beta$-环糊精(1)连接起来, 其中阿霉素/石墨烯和卟 啉/石墨烯之间都是通过超分子 $\pi-\pi$ 作用连接在一起，环 糊精通过对修饰在吓啉上的金刚烷基团产生包和作用 与之相连(图 4). 


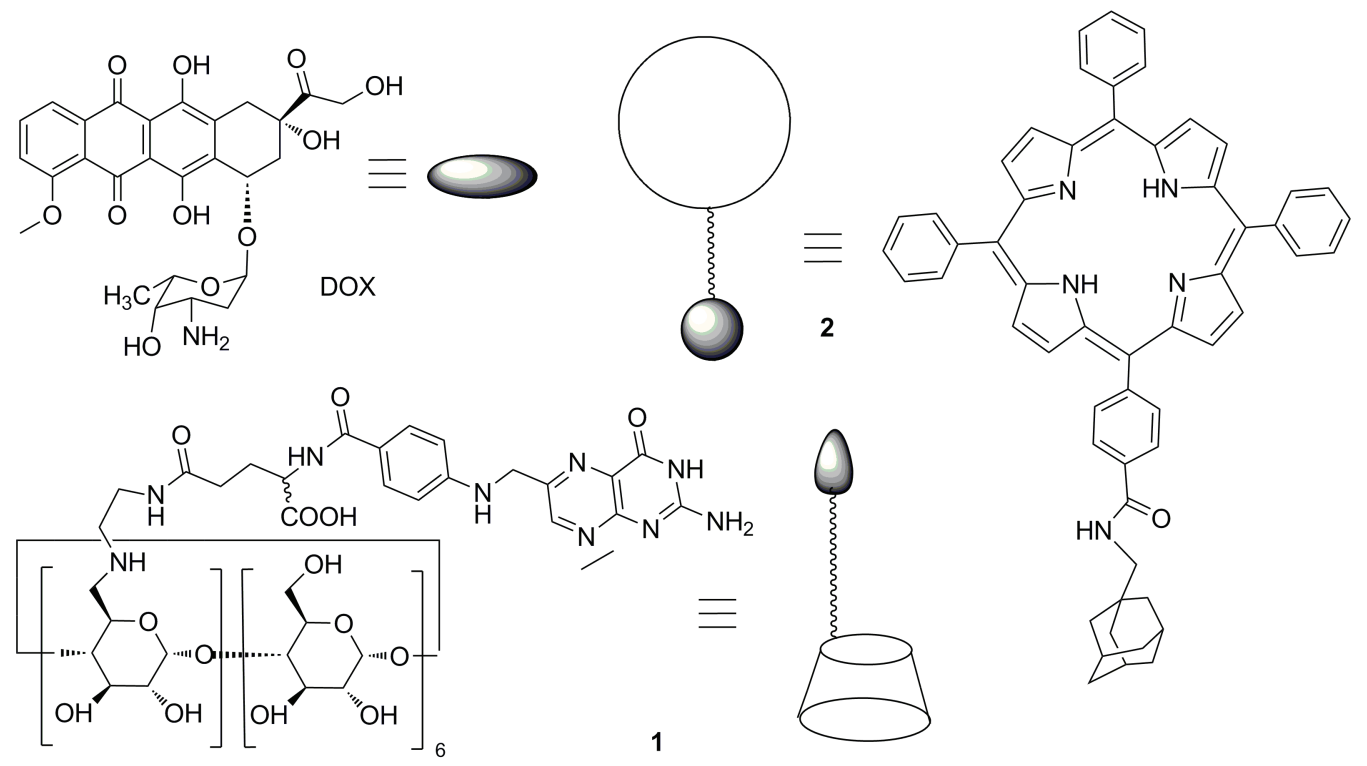

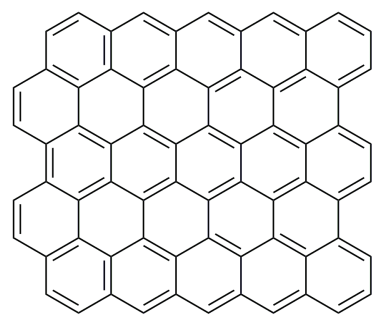

$\mathrm{GO}$
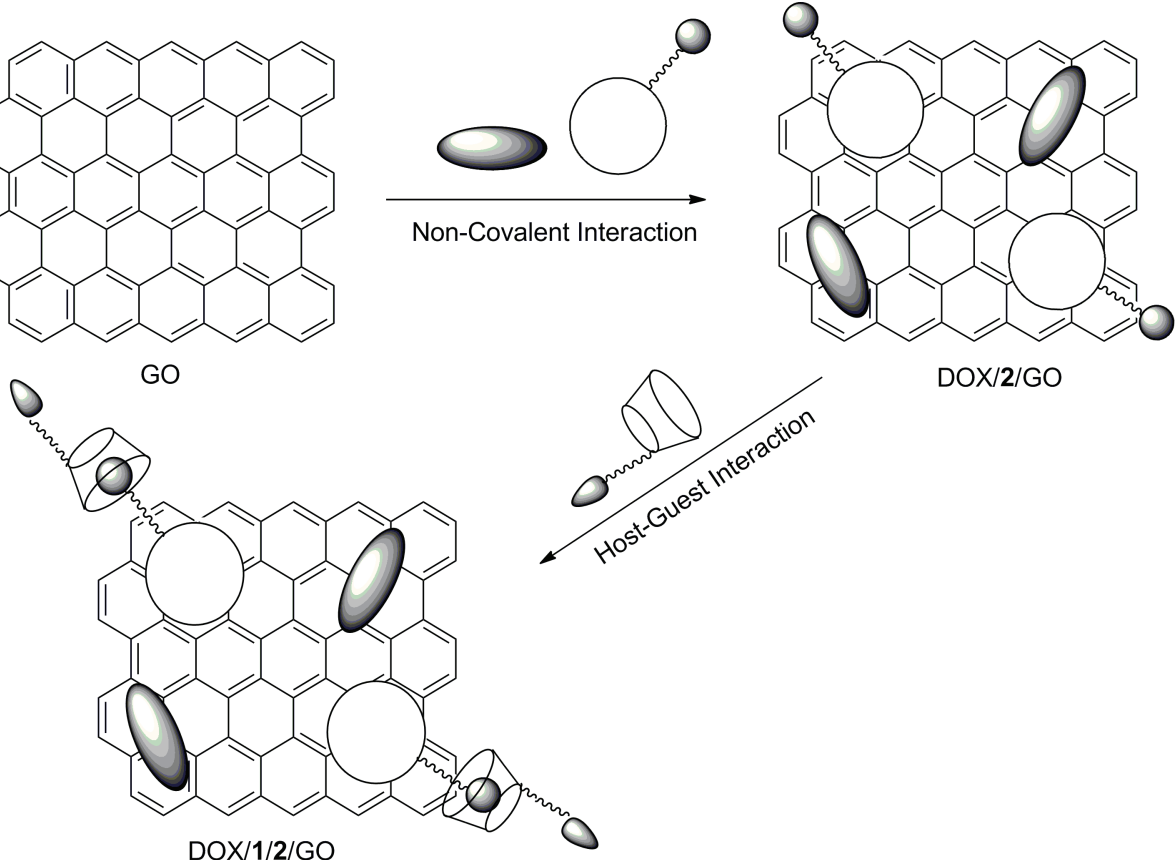

图 4 环糊精-石墨烯一卟啉超分子体系

Figure 4 Cyclodextrin-graphene-porphyrin system

Ogoshi 等 ${ }^{[31]}$ 仅通过离子作用和主客体作用等超分 子作用便得到了一种环糊精-石墨烯超分子体系(图 5). 利用全-6-氨基-脱氧 $-\beta$-环糊精的氨基与氧化石墨烯的 羧基之间的阴阳离子相互作用得到了环糊精-石墨烯超 分子体系, 并通过红外吸收光谱研究和证实了这种作 用. 利用固载在石墨烯上的环糊精的空腔的分子识别作 用, 分别对金刚烷羧酸钠 (AdCNa) 和一种双头金刚烷化 合物(Ad-Dimer)进行了识别和作用, 发现这种环糊精石墨烯超分子体系对二者均有较好的分子识别和包结 作用. 有趣的是, 双头金刚烷化合物一旦加入到此环糊 精-石墨烯超分子体系中会形成三维的类似聚合物的网
格结构, 并且通过动态光散射 (dynamic light scattering, DLS)研究发现该分散体系中的微观聚集体的尺寸会从 $500 \mathrm{~nm}$ 骤升至 $1500 \mathrm{~nm}$, 这是由于双头金刚烷化合物分 别进入不同环糊精空腔，起了不同构筑基元之间 “连接 桥” 的作用.

Ogoshi 等 ${ }^{[31}$ 提供的将电荷引入石墨烯的方法可能 会在某种程度上影响石墨烯的电化学性能, Han 等 ${ }^{[22]}$ 采 用了非静电作用的超分子作用来避免这种可能的结果. Han 等利用三嵌段共聚物[poly(ethylene oxide)-blockpoly(propylene oxide)-block-poly-(ethylene oxide) triblock copolymer, PEO- $b$-PPO- $b$-PEO]作为增溶剂, 通过三嵌段 


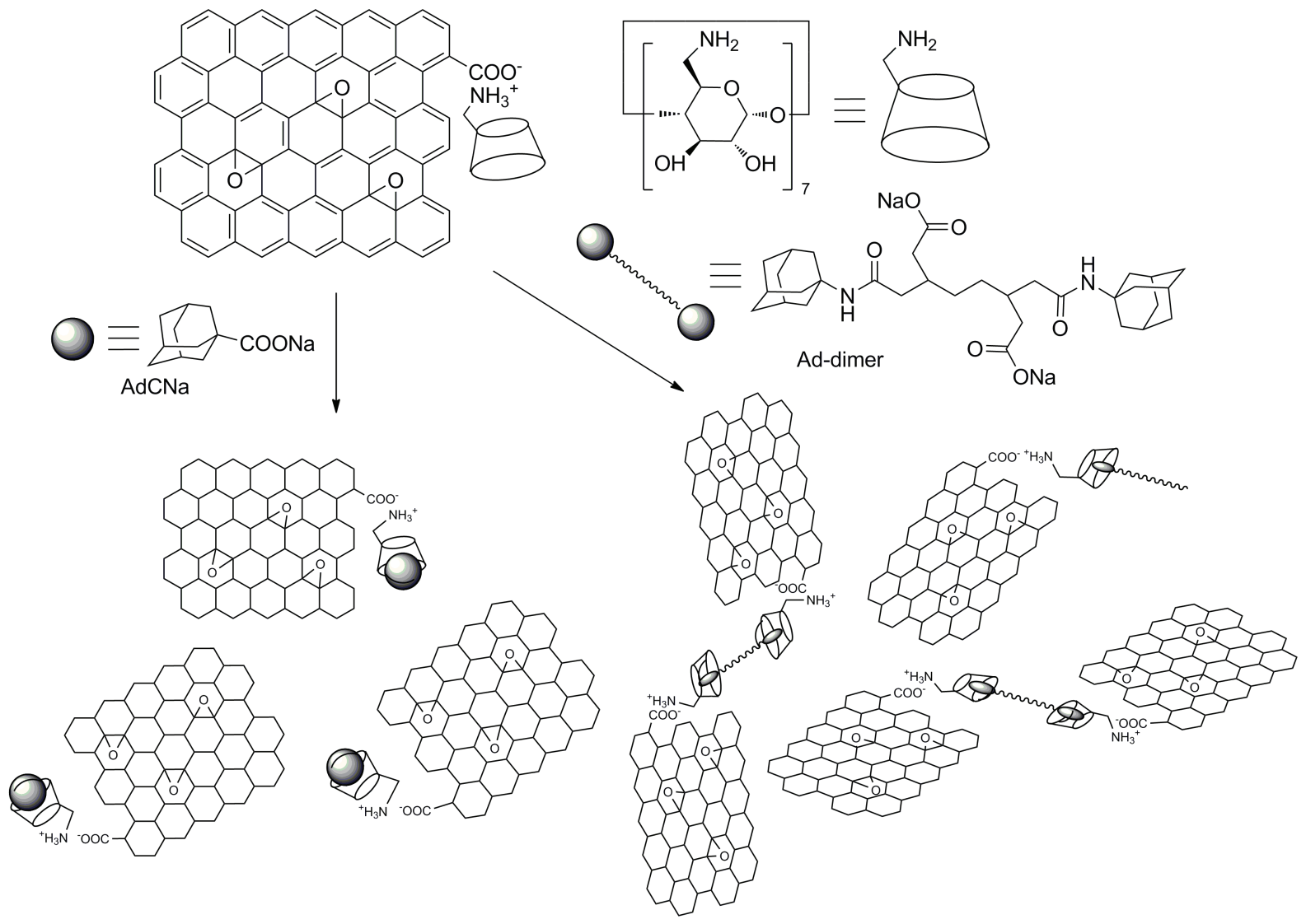

图 5 环糊精-石墨烯-金刚烷超分子体系

Figure 5 Cyclodextrin-graphene-adamantane system

共聚物中疏水的的 PPO 部分与同样疏水的石墨烯表面 发生疏水-疏水作用, 而 PEO 部分可以深入到水中, 使 三嵌段共聚物可以附着在石墨烯表面并使其可以稳定
分散到水溶液中(图 6). 已有报道称 PEO 与 $\alpha$-环糊精形 成的聚轮烷可形成超分子水凝胶 ${ }^{[30]}$. Han 等在此体系中 加入未修饰的 $\alpha$-环糊精, PEO 链部分可以 “串” 入到 $\alpha$ -

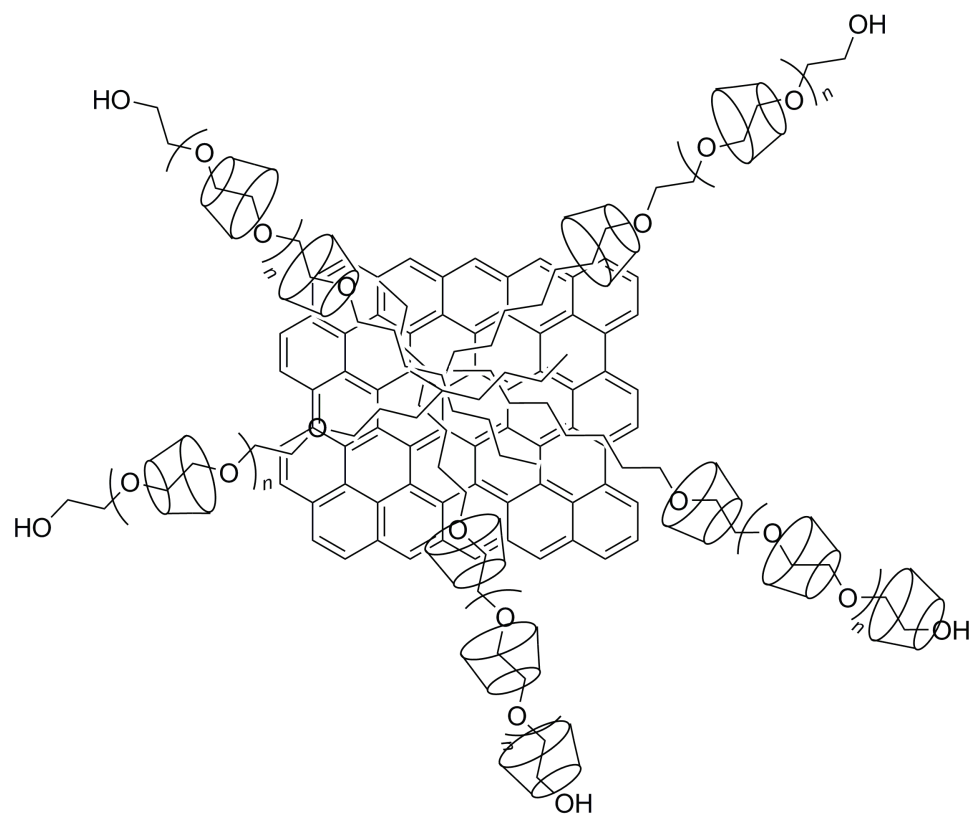

图 6 PEO 连接的环糊精-石墨烯超分子体系

Figure 6 Cyclodextrin-graphene system linked by PEO 
环糊精空腔内, 形成准聚轮烷的结构, 并使原石墨烯分 散溶液转化成对温度相应的凝胶体系. 温度升高, 可能 会导致 $\alpha$-环糊精与 PEO 的脱包和, 进而破坏空间网格 结构. Han 等特别指出, 与仅用 $\alpha$-环糊精与三嵌段共聚 物形成的凝胶相比, 由于石墨烯层状结构的存在, 这种 凝胶的粘度与强度有着明显的下降, 但溶胶一凝胶转化 时间缩短, 这是由于石墨烯能够梳理 PPO 部分, 避免其 自聚集成胶束所致. Han 等报道的环糊精-石墨烯超分子 体系构筑基元之间完全是通过超分子作用相连接, 是一 次值得肯定的有益尝试.

Dong 和 Wang 等 ${ }^{[34]}$ 与 Zhang 等 ${ }^{[35]}$ 也通过氧化石墨 烯/环糊精分散一还原一干燥的流程制备了在极性溶剂中 具有良好溶解性和稳定性的环糊精一石墨烯超分子体系, 并利用多种手段证实了每张石墨烯片上可以有效固定 多个环糊精分子. Dong 和 Wang 等尝试了 $\alpha-, \beta$-和 $\gamma$-环糊 精并都获得了成功. 他们提出的方法简便、迅速并有效, 并希望由此实现一种制备环糊精一石墨烯超分子体系的 通用方法. Wang 等 ${ }^{[34]}$ 还尝试了在环糊精-石墨烯超分子 体系修饰电极制备过程中加入了 Nafion 材料以增强电 极的机械性能.

Yuan 等 ${ }^{[37}$ 制备了聚环糊精-石墨烯一多层碳纳米管 超分子体系修饰的电极: 分别将多层碳纳米管与石墨烯 加入到 $\beta$-环糊精和聚 $\beta$-环糊精的水溶液中并进行超声 波分散 $1 \mathrm{~h}$ 得到稳定的黑色悬浊液, 然后滴到玻碳电极 上在空气中晾干得到修饰的电极. 经扫描电子显微镜 (scanning electron microscope, SEM)表征, 一维的碳纳
米管与二维的石墨烯在聚环糊精的作用下分散成了均 匀的三维空间网格状结构.

$\mathrm{Wu}$ 等 ${ }^{[38]}$ 通过电化学还原的方法, 在玻碳电极上修 饰了环糊精一石墨烯超分子体系: 超声条件下将氧化石 墨烯与 $\beta$-环糊精分散到水体系中, 然后滴到玻碳电极 上, 在浓度为 $0.1 \mathrm{~mol} / \mathrm{L}$ 的 $\mathrm{pH}=7.0 \mathrm{PBS}$ 缓冲溶液中、电 位 $-1.2 \sim+1.2 \mathrm{~V}$ 之间、扫描速率为 $100 \mathrm{mV} \cdot \mathrm{s}^{-1}$ 条件下 进行电化学还原, 水洗并用 $\mathrm{N}_{2}$ 干燥后得到环糊精一石墨 烯超分子体系修饰的玻碳电极.

贵金属材料通常具有良好的化学物理性质，被誉为 “现代材料的维生素”. 金材料具有惊人的延展性、优秀 的电催化活性、良好的电传导性及生物相容性，可应用 于光学、电化学、传感器、燃料电池等许多领域 ${ }^{[39]}$. 将 金材料, 特别是金纳米材料引入到环糊精一石墨烯超分 子体系中, 可能会产生意想不到的效果. Choi 等 ${ }^{[40]}$ 将氧 化石墨烯、 $\beta$-环糊精及氯金酸 $\left(\mathrm{HAuCl}_{4}\right)$ 均匀混合并在碱 性条件下加热处理 $2 \mathrm{~h}$, 经离心、洗涤、干燥等过程得到 纳米金(Au nanoparticles, AuNP)-环糊精-石墨烯三元体 系(图 7). 在这个三元体系的制备过程中, 环糊精其实起 了还原剂的作用.

Chen 等 ${ }^{[41]}$ 在环糊精-石墨烯体系中引入了另一种 贵金属铂. 氧化石墨烯、 $\beta$-环糊精及氯铂酸 $\left(\mathrm{H}_{2} \mathrm{PtCl}_{6}\right)$ 加 热条件下经肼的氨水溶液还原，并经过滤、洗涤、干燥 得到纳米铂(Pt nanoparticles, PtNP)-环糊精-石墨烯三元 体系.

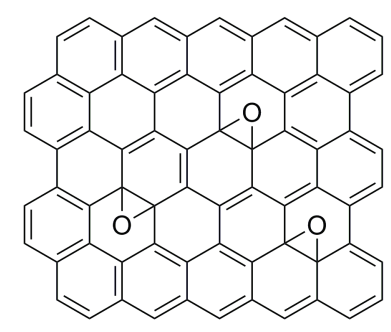

$\mathrm{GO}$

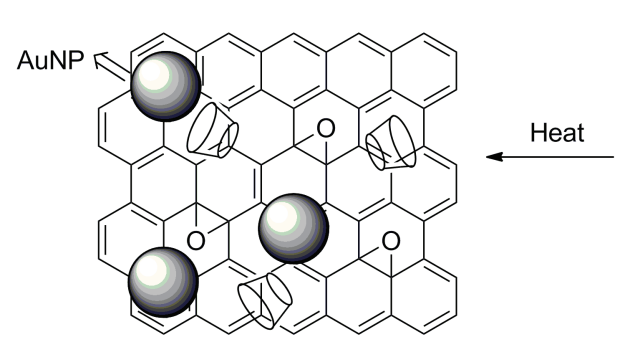

图 7 负载有纳米金的环糊精一石墨烯超分子体系的制备过程

Figure 7 Preparation of cyclodextrin-graphene system loaded with AuNP 


\section{2 环糊精一石墨烯超分子体系的应用}

\section{1 药物运输}

由于叶酸修饰的 $\beta$-环糊精可以识别癌细胞中的叶 酸受体. Liu 等 ${ }^{[30]}$ 报道了通过上文提到的复杂的四元超 分子体系通过各个部分之间的协同作用，相对于游离的 阿霉素来说, 在有机体内有着更好的抗癌活性和更低的 机体毒性. 值得一提的是, 叶酸修饰的 $\beta$-环糊精分子存 在自包和现象, 即叶酸部分会进入环糊精空腔内部, 但 金刚烷会将叶酸取代出来, 这是由于金刚烷与 $\beta$-环糊精 有着更大的包和常数所致.

\section{2 电化学检测}

\subsection{1 对药物分子的检测}

环糊精一石墨烯超分子体系除了可以用来运输抗癌 药物之外 ${ }^{[30]}$, 可以用来对药物的电化学检测. Dong 等 ${ }^{[22]}$ 利用环糊精一石墨烯超分子体系修饰的玻碳电极对抗癌 药物阿霉素(adriamycin)和甲氨蝶呤(methotrexate)(图 8) 进行了电化学检测. 检测电流的峰值相对于裸玻碳电极 分别提高了 26.5 和 23.7 倍, 二者的检出限分别为 0.1 和 $20 \mathrm{nmol} / \mathrm{L}$, 且均有良好的重现性. 阿霉素和甲氨蝶呤有 着较大的检出限差异可能与环糊精对二者的分子识别 能力的差异有关.

Dong 和 Wang 等 ${ }^{[34]}$ 尝试了利用环糊精一石墨烯超分 子体系修饰的电极对 8 种生物分子或药物分子进行电化 学检测, 发现检测效果均优于未修饰的石墨烯或碳纳米 管, 这可能是与石墨烯(高电传导效率与高比表面积)和 环糊精(超分子识别与富集性能)的协同作用有关.

Wang 等 ${ }^{[34]}$ 利用环糊精一石墨烯超分子体系实现了 对芸香式的低浓度灵敏检测, 检出限可低至 $2 \times 10^{-9}$ $\mathrm{mol} / \mathrm{L}$.

\subsection{2 对污染物的检测}

Wang 等 ${ }^{[24]}$ 利用微波催化法制备的环糊精-石墨烯 超分子体系也可修饰在玻碳电极上, 并可以检测某些特 定的苯系污染物分子, 其中对硝基苯酚的检出限低至 $10^{-8} \mathrm{~mol} / \mathrm{L}$.
Chen 等 ${ }^{[41]}$ 发展的纳米铂(PtNP)-环糊精-石墨烯三 元体系可用于电化学检测环境污染物萘酚, 并对其不同 的异构体 ( $\alpha$-荟酚、 $\beta$-荟酚)有着不同的检出值, 这可能也 是与环糊精的分子识别性能相关.

$\mathrm{Wu}$ 等 ${ }^{[38]}$ 利用环糊精-石墨烯超分子体系修饰的玻 碳电极对甲基对硫磷等有机磷杀虫剂进行了电化学检 测，观测到甲基对硫磷可在这种电极上迅速富集. 这种 新型电极表现出了高灵敏性、高选择性与快速响应性的 优点. 最低检出限低至 $0.05 \times 10^{-9} \mathrm{~mol} / \mathrm{L}$, 灵敏度相对 于其他常用电极提高了 10 倍左右. 无疑, 环糊精的分子 识别性能起了重要作用, 这为检测痕量残留的有机磷农 药提供了新途径.

Dong 等 ${ }^{[43]}$ 应用环糊精-石墨烯超分子体系对一种 常用的广谱杀菌剂多菌灵进行了电化学检测. 石墨烯修 饰的玻碳电极和环糊精一石墨烯超分子体系修饰的玻碳 电极的检测能力分别比裸玻碳电极提高了 11.7 和 82.0 倍, 这也体现出了引入环糊精识别性能的重要性. 多菌 灵的检出限为 $2 \mathrm{nmol} / \mathrm{L}$, 其中浓度在 $5 \sim 0.45 \mu \mathrm{mol} / \mathrm{L}$ 之 间时，检出电流与浓度有良好的线性关系. 从上述这两 个例子可以看出，基于环糊精一石墨烯超分子体系的电 化学检测方法有望发展成为对某些易残留污染物分子 的常规检测手段.

\subsection{3 对生物分子的检测}

Wang 等 ${ }^{[29]}$ 还制备了稳定的环糊精-石墨烯氧化物卟啉玻碳电极, 并研究了电极上卟啉与血红蛋白的电化 学相关作用, 并得到了 $5.0 \times 10^{-9} \mathrm{~mol} / \mathrm{L}$ 的最低检测限, 这也表明其具有高效的生物学识别和检测能力. 但 Wang 等偏重于利用荧光吸收光谱、紫外-可见吸收光谱 和循环伏安法等手段检测体系性质，未提及关于卟啉在 环糊精-石墨烯体系中具体所处位置和状态.

多巴胺作为一种重要的神经传递介质, 在中枢神经 系统、肾脏、荷尔蒙分泌以及心血管功能过程中起着重 要作用 ${ }^{[44]}$. 对多巴胺的检测在诊断和治疗多巴胺代谢异 常类疾病(如癫㾁、老年痴呆症、帕金森症、精神分裂症
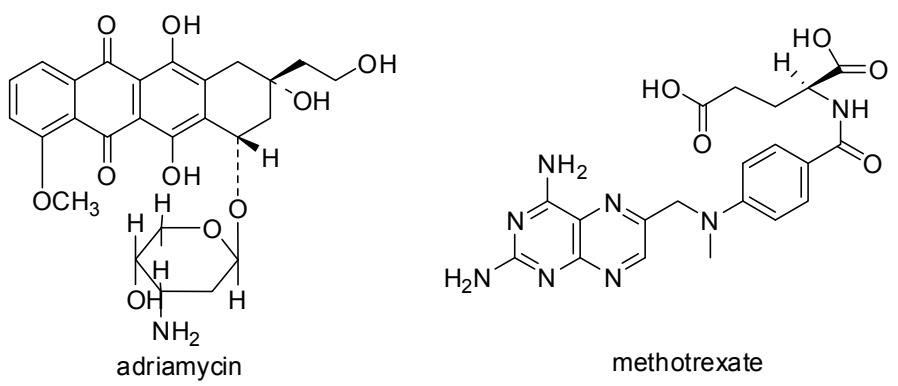

图 8 阿霉素与甲氨蝶呤结构图

Figure 8 Structures of adriamycin and methotrexate 
及艾滋病等)中有着重要意义 ${ }^{[45,46]}$. 但是在对多巴胺检 测过程中一个重要问题就是许多伴生的干扰化合物, 包 括抗坏血酸和亚硝酸根离子. 而 Yuan 等发展的聚环糊 精-石墨烯-多层碳纳米管超分子体系修饰的电极更是 实现了对这三种物质的同时检测. Yuan 等 ${ }^{[37]}$ 分析这是 由于碳纳米管阻碍了石墨烯的自堆积, 提高了其电化学 性能, 同时聚环糊精提高了碳纳米管-石墨烯的分散度 和溶解度, 环糊精的空腔在识别多巴胺与抗坏血酸过程 中也起了重要作用.

Zhang 等 ${ }^{[35]}$ 报道的环糊精一石墨烯超分子体系修饰 的电极也可用于多巴胺的检测, 并具有低检出值、宽线 性范围、抗抗坏血酸干扰的优点. 多巴胺在此类电极上 的反应显示是一种较广的扩散控制过程, 这与未经环糊 精修饰的石墨烯电极是不同的.

Choi 等 ${ }^{[40]}$ 制备的纳米金(AuNP)-环糊精一石墨烯三 元体系修饰的玻碳电极同样可以在多巴胺、抗坏血酸、 尿酸同时存在时实施电化学检测, 三者的检出限分别为 $0.15,10,0.21 \mu \mathrm{mol} / \mathrm{L}$.

\section{3 对环糊精-石墨烯超分子体系的前景展望}

目前环糊精一石墨烯超分子体系已经在很多领域显 现出巨大的潜在应用价值, 特别是在电化学分析领域有 许多革命性的突破. 鉴于环糊精空腔大小的限制 $(\alpha-C D$ : $5.3 \AA ; \beta$-CD: $6.5 \AA ; \gamma$-CD: $8.3 \AA)^{[47]}$, 其分子识别性能目 前还仅限于尺寸合适的客体无机或有机小分子. 如果将 基于环糊精的分子印迹技术 ${ }^{[48]}$ 引入到环糊精-石墨烯超 分子体系中来, 将突破环糊精空腔大小对客体分子选择 的限制, 这将特别在某些 “巨型” 生物分子的电化学检 测领域有应用空间. 另外, 超分子化学本身就是一门交 叉学科, 环糊精一石墨烯超分子体系更是在许多交叉领 域有着潜在应用前景.

\section{1 药物负载及释放}

延长人类的生命、提高人类的生活质量是人们永恒 的追求. 石墨烯是一类仅由碳原子构筑的新型材料, 而 环糊精则是由葡萄糖单元组成的环状结构, 二者均是生 物相容性良好的材料, 因此环糊精一石墨烯超分子体系 在作为药物的载体, 在特定药物的装载、运输、靶向释 放或按需释放过程中将有更广阔的应用. 关于环糊精装 载药物的例子数不胜数, 石墨烯负载药物也有优秀的先

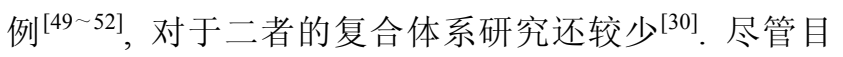
前在此领域的研究才刚起步, 但已展示出了广阔的前 景. 考虑到环糊精与石墨烯均能负载药物, 但二者与药 物的作用机制不尽相同, 因此我们可以设想将不同种类 的药物负载在同一个环糊精-石墨烯超分子体系中, 控 制不同的外界条件或对外界不同环境进行刺激响应, 有
可能达到药物按需分级释放的结果, 这将是一个诱人的 领域.

\section{2 模拟生物固氮}

$\gamma$-环糊精与富勒烯的包和超分子体系曾因在温和条 件下 $\left(101 \mathrm{kPa} \mathrm{N}_{2}, 60{ }^{\circ} \mathrm{C}, \mathrm{h} v\right)$ 模拟生物固氮而名噪一时 ${ }^{[53]}$. 石墨烯具有良好的导电性能, 且仅由一层原子构成, 是 目前已知最薄的材料, 相对于富勒烯有着更大的比表面 积和更多的作用位点，因此设想环糊精一石墨烯超分子 体系可能在类似模拟生物固氮作用等领域有着潜在应 用, 这在能源与资源日趋捉襟见肘的今天显得尤为重 要.

\section{3 新型燃料电池}

环糊精空腔具有良好的分子识别性能, 并且环糊精 空腔具有富电性, 作为纳米反应器可以催化一系列的化 学反应 ${ }^{[15]}$. 如果将环糊精空腔看做良好的分子捕捉与 加工位点, 并利用石墨烯的良好的电子传导与转移性 能, 可能会实现新型、高效、无污染的燃料电池的制备, 这将在合理利用资源保护生态环境等领域有着深远的 意义.

\section{4 研究电子传导过程}

光诱导的电子传导 (photoinduced electron-transfer, PET), 在光驱动分子器件、解释光合作用、光能开发利 用等领域应用广泛，一直是目前化学界与生物界研究的 热点问题. 四硫富瓦烯(tetrathiafulvalene, TTF)是富瓦烯 的 2,2'-位被硫原子替换后形成的化合物, 是强 $\pi$ 电子供 体 ${ }^{[51]}$. Liu 等 ${ }^{[55]}$ 曾利用环糊精一富勒烯体系与水溶性卟啉 之间的自组装而形成特殊纳米线型聚集体研究了光诱 导的电子传导过程. 考虑到石墨烯具有的较大的作用面 积和环糊精具有的分子识别性能, 如果在环糊精-富勒 烯体系引入四硫富瓦烯或前文已介绍过的卟啉等电子 供体, 将在人工模拟光合作用等领域有潜在应用价值.

\section{4 结论}

我国科学家在发展环糊精一石墨烯超分子体系领域 已经走在了世界研究的前沿. 这个领域方兴未艾, 函待 超分子化学、电化学、材料学、生命科学等各个领域的 专家学者的继续努力. 在面对能源、资源、环境危机并 强调可持续发展的当今社会, 环糊精一石墨烯超分子体 系以其独特而优秀的性能必将受到越来越多的关注.

\section{References}

[1] Xu, J,-Y. Chin. J. Org. Chem. 1995, 15, 133 (in Chinese). (徐家业, 有机化学, 1995, 15, 133.)

[2] Pedersen, C. J. Angew. Chem., Int. Ed. Engl. 1988, 27, 1021.

[3] Cram, D. J. Angew. Chem., Int. Ed. Engl. 1988, 27, 1009, 
[4] Lehn, J. M. Angew. Chem., Int. Ed. Engl. 1988, 27, 89.

[5] Novoselov, K. S.; Geim, A. K.; Firsov, A. A. Science 2004, 306, 666.

[6] Zhang, Y.-B.; Tan, Y.-W.; Stormer, H. L.; Kim, P. Nature 2005, 438,201 .

[7] Gomez-Navarro, C.; Weitz, R. T.; Bittner, A. M.; Scolari, M.; Mews, A.; Burghard, M.; Kern, K. Nano Lett. 2007, 7(11), 3499.

[8] Rutter, G. M.; Crain, J. N.; Guisinger, N. P.; Li, T.; First, P. N.; Stroscio, J. A. Science 2007, 317, 219.

[9] Oostinga, J. B.; Heersche, H. B.; Liu, X. L.; Morpurgo, A. F.; Vandersypen, L. M. K. Nat. Mater. 2008, 7(2), 151.

[10] Balandin, A. A.; Ghosh, S.; Bao, W. Z.; Calizo, I.; Teweldebrhan, D.; Miao, F.; Lau, C. N. Nano Lett. 2008, 8(3), 902.

[11] Dikin, D. A.; Stankovich, S.; Zimney, E. J.; Piner, R. D.; Dommett, G. H. B.; Evmenenko, G.; Nguyen, S. T.; Ruoff, R. S. Nature 2007, 448, 457.

[12] Park, S.; Lee, K. S.; Bozoklu, G.; Cai, W.; Nguyen, S. T.; Ruoff, R. S. ACS Nano 2008, 2(3), 572.

[13] Stankovich, S.; Dikin, D. A.; Dommett, G. H. B.; Kohlhaas, K. M.; Zimney, E. J.; Stach, E. A.; Piner, R. D.; Nguyen, S. T.; Ruoff, R. S. Nature 2006, 442, 282.

[14] Tong, L.-H. Cyclodextrin Chemistry - Foundation and Application, Science Press, Beijing, 2001, pp. 10 20 (in Chinese). (童林荟, 环糊精化学——基础与应用, 科学出版社, 北京 2001, pp. 10 20.)

[15] Sun, T.; Hao, A.-Y.; Shen, J. Chin. J. Org. Chem. 2009, 29(5), 681 (in Chinese).

(孙涛, 郝爱友, 申健, 有机化学, 2009, 29(5), 681.)

[16] Sun, T.; Zhang, H.-C.; Kong, L.; Qiao, H.-W.; Li, Y.-M.; Xin, F.-F.; Hao, A.-Y. Carbohydr. Res. 2011, 346, 285.

[17] Chen, S.-Y.; Zhou, X.-T.; Ji, H.-B. Chin. J. Org. Chem. 2012, 32(4), 686 (in Chinese). (陈韶云, 周贤太, 纪红兵, 有机化学, 2012, 32(4), 686.)

[18] Chen, Y.; Liu, Y. Chin. J. Org. Chem. 2012, 32(5), 805 (in Chinese). (陈涌, 刘育, 有机化学, 2012, 32(5), 805.)

[19] Zhou, D.-X.; Sun, T.; Deng, W. Chin. J. Org. Chem. 2012, 32(2), 239 (in Chinese). (周冬香, 孙涛, 邓维, 有机化学, 2012, 32(2), 239.)

[20] Sun, H.-Y.; Bai, Y.; Zhao, M.-G.; Hao, A.-Y.; Xu, G.-Y.; Shen, J.; Li, J.-Y.; Sun, T.; Zhang, H.-C. Carbohydr. Res. 2009, 344(15), 1999.

[21] Zhang, H.-C.; Liu, Z.-N.; Xin, F.-F.; An, W.; Hao, A.-Y.; Li, J.-Y.; Li, Y.-Y.; Sun, L.-Z.; Sun, T.; Zhao, W.-J.; Li, Y.-M.; Kong, L. Carbohydr. Res. 2011, 346(2), 294.

[22] Jin, Z.-Y.; Xu, X.-M.; Chen, H.-Q.; Li X.-B. Cyclodextrin Chemistry, Chemical Industry Press, Beijing, 2009 (in Chinese). (金征宇, 徐学明, 陈寒青, 李学红, 环糊精化学, 化学工业出版 社, 北京, 2009.)

[23] Liu, J.-H.; Chen, G.-S.; Jiang, M. Macromolecules 2011, 44, 7682.

[24] Xu, C.-H.; Wang, J.-C.; Wan, L.; Lin J.-J.; Wang, X.-B. J. Mater. Chem. 2011, 21, 10463

[25] Baskaran, D.; Mays, J. W.; Zhang, X. P.; Bratcher, M. S. J. Am. Chem. Soc. 2005, 127, 6916.

[26] Guldi, D. M.; Rahman, G. M. A.; Jux, N.; Balbinot, D.; Hartnagel, U.; Tagmatarchis, N.; Prato, M. J. Am. Chem. Soc. 2005, 127, 9830.

[27] Guldi, D. M.; Rahman, G. M. A.; Jux, N.; Tagmatarchis, N.; Prato, M. Angew. Chem., Int. Ed. 2004, 43, 5526.

[28] Guldi, D. M.; Taieb, H.; Rahman, G. M. A.; Tagmatarchis, N.;
Prato, M. Adv. Mater. 2005, 17, 871.

[29] Xu, C.-H.; Wang, X.-B.; Wang, J.-C.; Hu, H.-T.; Wan, L. Chem. Phys. Lett. 2010, 498, 162.

[30] Yang, Y.; Zhang, Y.-M.; Chen, Y.; Zhao, D.; Chen, J.-T.; Liu, Y. Chem. Eur. J. 2012, 18, 4208.

[31] Ogoshi, T.; Ichihara, Y.; Yamagishi, T.; Nakamoto, Y. Chem. Commun. 2010, 46, 6087.

[32] Zu, S.-Z.; Han, B.-H. J. Phys. Chem. C 2009, 113, 13651.

[33] Wenz, G.; Han, B.-H.; Müller, A. Chem. Rev. 2006, 106(3), 782.

[34] Guo, Y,-J.; Guo, S.-J.; Ren, J.-T.; Zhai, Y.-M.; Dong, S.-J.; Wang, E.-K. Acs Nano 2010, 4(7), 4001.

[35] Tan, L.; Zhou, K.-G.; Zhang, Y.-H.; Wang, H.-X.; Wang, X.-D.; Guo, Y.-F.; Zhang, H.-L. Electrochem. Commun. 2010, 12, 557.

[36] Liu, K.-P.; Wei, J.-P.; Wang, C.-M. Electrochim. Acta 2011, 56, 5189.

[37] Zhang, Y.; Yuan, R.; Chai, Y.-Q.; Li, W.-J.; Zhong, X.; Zhong, H.-A. Biosens. Bioelectron. 2011, 26, 3977.

[38] Wu, S.; Lan, X.-Q.; Cui, L.-J.; Zhang, L.-H.; Tao, S.-Y.; Wang, H.-N.; Han, M.; Liu, Z.-G.; Meng C.-G. Anal. Chim. Acta 2011, 699, 170.

[39] Stender, A. S.; Wang, G. F.; Sun, W.; Fang, N. ACS Nano 2010, 4, 7667.

[40] Tian, X.-Q.; Cheng, C.-M.; Yuan, H.-Y.; Du, J.; Xiao, D.; Xie, S.-P.; Choi, M. M. F. Talanta 2012, 93, 79.

[41] Zhu, G.-B.; Gai, P.-B.; Wu, L.; Zhang, J.-H.; Zhang, X.-H.; Chen, J.-H. Chem. Asian J. 2012, 7, 732.

[42] Guo, Y.-J.; Chen, Y.-H.; Zhao, Q.; Shuang, S.-M.; Dong, C. Electroanal. 2011, 23(10), 2400.

[43] Guo, Y.-J.; Guo, S.-J.; Li, J.; Wang, E.-K.; Dong, S.-J. Talanta 2011, 84, 60 .

[44] Heien, M. L. A. V.; Khan, A. S.; Ariansen, J. L.; Cheer, J. F.; Phillips, P. E. M.; Wassum, K. M.; Wightman, R. M. Proc. Natl. Acad. Sci. U. S. A. 2005, 102, 10023.

[45] Ali, S. R.; Ma, Y. F.; Parajuli, R. S. R.; Balogun, Y.; Lai, W. Y. C.; He, H. X. Anal. Chem. 2007, 79, 2583.

[46] Zhou, X.; Zheng, N.; Hou, S. R.; Li, X. J.; Yuan, Z. B. J. Electroanal. Chem. 2010, 642, 30.

[47] Sun, T.; Li, Y.-M.; Xin, F.-F.; Li, S.-Y.; Hou, Y.-H.; Hao, A.-Y. Prog. Chem. 2012, 24, 71 (in Chinese).

(孙涛, 李月明, 辛飞飞, 李尚洋, 侯月会, 郝爱友, 化学进展, 2012, 24, 71.)

[48] Sun, T.; Li, Y.-Y.; Zhang, H.-C.; Hao, A.-Y. Prog. Chem. 2010, 22, 888 (in Chinese). (孙涛, 李媛媛, 张华承, 郝爱友, 化学进展, 2010, 22, 888.)

[49] Liu, Z.; Sun, X.; Nakayama-Ratchford, N.; Dai, H. ACS Nano 2007, 1, 50 .

[50] Liu, Z.; Robinson, J. T.; Sun, X.; Dai, H. J. Am. Chem. Soc. 2008, 130, 10876.

[51] Dhar, S.; Liu, Z.; Thomale, J.; Dai, H.; Lippard, S. J. J. Am. Chem. Soc. 2008, 130, 11467.

[52] Sun, X.; Liu, Z.; Welsher, K.; Robinson, J. T.; Goodwin, A.; Zaric, S.; Dai, H. Nano Res. 2008, 1, 203.

[53] Nishibayashi, Y.; Saito, M.; Uemura, S.; Takekuma, S.; Takekuma, H.; Yoshida, Z. Nature 2004, 428, 279.

[54] Giacalone, F.; D'Anna, F.; Giacalone, R.; Gruttadauria, M.; Riela, S.; Noto, R. Tetrahedron Lett. 2006, 47, 8105.

[55] Zhang, Y.-M.; Chen, Y.; Yang, Y.; Liu, P.; Liu, Y. Chem. Eur. J. 2009, 15, 11333 . 